\title{
La interculturalidad en el Ecuador: un análisis crítico de sus fundamentos, aportes y desafíos en la educación
}

\author{
Juan Gabriel Aceldo Rodríguez \\ juanaceldor@gmail.com \\ ORCID ID: 0000-0002-5739-6195 \\ Luis Miguel Quito Suco \\ luismiguelquito123@gmail.com \\ ORCID ID: 0000-0002-9283-6686 \\ Universidad Católica de Cuenca, UCACUE \\ Cuenca - Ecuador
}

\section{RESUMEN (200)}

El presente trabajo responde a un ensayo crítico de la interculturalidad en el Ecuador desde el campo educativo, para efecto se parte de la conceptualización de las terminologías; Interculturalidad, Pluriculturalidad y Multiculturalidad, mismas que han sido usado para definir esa interrelación entre culturas. A partir de esa conceptualización se hace una reflexión crítica y se da a conocer porque en la actualidad es más usado el término de intercultural para describir al estado ecuatoriano, todo esto argumentado con aportes de varios autores y organizaciones que analizan la interculturalidad que hoy en día se vive, y que por las diversas reformar educativas poco a poco se las pone en práctica en las aulas de clase. Por ende, se realiza un análisis desde las reformas curriculares y como éstas ayudan al desarrollo de la convivencia armoniosa entre los diferentes grupos sociales que en la actualidad interactúan en un mismo entorno social, además de los desafíos que se han identificado con la inserción de la interculturalidad en la educación del Ecuador.

Palabras clave: interculturalidad; pluriculturalidad; multiculturalidad; educación; ecuador. 


\title{
Interculturality in Ecuador: a critical analysis of its foundations, contributions and challenges in education
}

\begin{abstract}
The present work responds to a critical essay of the interculturality in Ecuador from the educational field, for effect it is part of the conceptualization of the terminologies; Interculturality, Pluriculturality and Multiculturalism, which have been used to define this interrelation between cultures. From this conceptualization a critical reflection is made and it is made known because at present the term intercultural is more used to describe the Ecuadorian state, all this argued with contributions from various authors and organizations that analyze the interculturality that today it is lived, and that by the diverse educational reforms little by little they are put into practice in the classrooms. Therefore, an analysis is carried out from the curricular reforms and how they help the development of harmonious coexistence between the different social groups that currently interact in the same social environment, in addition to the challenges that have been identified with the insertion of the interculturality in education in Ecuador.
\end{abstract}

Keywords: interculturality, pluriculturality, multiculturalism, education. Ecuador.

Artículo recibido: 05 octubre. 2021 Aceptado para publicación: 02 noviembre 2021

Correspondencia: juanaceldor@gmail.com Conflictos de Interés: Ninguna que declarar 


\section{INTRODUCCIÓN}

En el presente texto se analiza los diferentes conceptos que están inmersos dentro de la educación intercultural tomando como referencia los criterios de autores que abordan estas conceptualizaciones desde enfoque más cercano y experimentado sobre las la interculturalidad que en la actualidad se vive. Hay que tener en cuenta que la interculturalidad desde un enfoque educativo, con el paso del tiempo a progresado y ha sido tomada en cuenta con mayor ímpetu, dejando atrás creencias populares sobre las diferentes interpretaciones de la interculturalidad. Además, se analiza los aportes que brinda este progreso, así como los desafíos que conlleva el mismo.

Como punto de partida se detalla las diferentes concepciones sobre la interculturalidad, pluriculturalidad y multiculturalidad, además se da a conocer algunas de sus características desde un enfoque actual. Luego de esto se analizan estas conceptualizaciones inmersas ya en el campo educativo, interpretándolas desde las reformas educativas que han surgido hasta la actualidad.

Por tanto, el presente trabajo pretende realizar una discusión entre la interculturalidad y el contexto educativo partiendo desde el cómo es empleado el currículo, es decir, analizar cómo se ha ido aplicando estas concepciones y entrelazando una con otra en los diferentes niveles de educación. Finalmente se debate sobre las reformas educativas surgidas, a partir de la necesidad de interrelacionarse varias culturas o grupos étnicos en una misma sociedad en desarrollo, sea estos indígenas, afro ecuatorianos, shuar y demás grupos étnicos, por lo cual es de vital importancia establecer un contexto de convivencia con un ambiente de armonía y sobre todo de forma íntegra en la sociedad.

\section{DESARROLLO}

Con el paso del tiempo en Ecuador se han ido concretando varias ideas y concepciones entorno a la interculturalidad y sus variantes plurales, mismas que deben ser analizadas para llegar a concluir, cuál sería la terminología apropiada para definir a este país rico en culturas. Por lo tanto, analizaremos independientemente las diferentes percepciones de los autores sobre la interculturalidad, pluricultural y multicultural, para dar a conocer como estos términos llegan a ser actualmente uno solo y en función de esta concepción analizar su nivel de inserción en la educación.

\section{Interculturalidad}

Con referencia al término interculturalidad se puede mencionar que es aquello que hace 
referencia a la pluralidad de culturas que conviven, se interrelacionan y comparten en determinado contexto y localidad. La RAE define a la interculturalidad como concerniente "a la relación entre culturas". Por lo cual, se entiende que este término engloba a toda situación de relación que existe entre dos o más culturas, sin preponderarse una de la otra, puesto que todos poseen los mismos derechos y obligaciones constitucionales. En concordancia con lo mencionado la UNESCO (s.f.) define la interculturalidad como "la presencia e interacción equitativa de diversas culturas y la posibilidad de generar expresiones culturales compartidas, adquiridas por medio del diálogo y de una actitud de respeto mutuo" (párr. 1).

En tal sentido la interculturalidad es un tema que conlleva un análisis consiente sobre la interrelación cultural existente, puesto que no se refiere al simple hecho de que dos o más cultural coincidan en un lugar o espacio físico, sino comprender las diferencias de cada una de ellas, aprender de ellas y sobre todo convivir ente sí, sin embargo, muchas de las veces las culturas no han querido o podido interrelacionarse de buena manera.

Podría afirmarse que el problema de la interculturalidad ha surgido como resultado de la profundización-discusión del problema de la cultura. Pues, a partir de la noción matriz de cultura se ha desarrollado una constelación terminológica que depende de ella. A guisa de ejemplo se pueden mencionar nociones como: culturas, aculturación, inculturación, pluriculturalidad, multiculturalidad e interculturalidad. (Higuera \& Castillo, 2015, p. 4).

Por lo tanto, se puede concluir que se denomina interculturalidad a las diferentes culturas agrupadas de manera que todas coexistan y respetándonos entre sí. Ahora bien, por tratarse de una nueva forma de comprender el concepto de interculturalidad podría resultar conflictiva y hasta ambigua tanto es así que, en algunos casos, cuando se anuncia la defensa o el impulso de la interculturalidad, lo que se pone en práctica coincide más a una promoción multicultural. Por ello, resulta imperioso definir algunos de los términos de la aludida constelación de cultura y coincidir en una terminología que realmente demuestre la realidad social del estado ecuatoriano.

\section{Pluriculturalidad}

Por su parte la pluriculturalidad consiste en la constatación de la existencia de dos o más 
culturas dentro de un mismo territorio y su posible interrelación, afirmándose, la importancia de su reconocimiento y su valoración en igualdad de condiciones. Bernabé (2012) considera que "el pluralismo cultural debe defenderse como categoría de toda sociedad democrática, como existencia de muchas culturas en un mismo territorio, defendiéndose el reconocimiento del otro y la igualdad" (p. 69).

De esta manera se puede reconocer la concepción del término pluricultural que en la constitución se conoce cómo el Sumak Kawsay, en donde se reconoce a indígenas y no indígenas, montuvios o no, shuar o no, entre otros. En definitiva, Ecuador es un país reconocido y se lo descubre como un país que posee muchas culturas dentro de su territorio, y fue incorporado en el 2008 dentro de nuestra constitución como principio de la nación.

En resumen, el terminó pluricultural es hace referencia de manera específica a reconocer que en un país existen varias culturas y que las mismas poseen, derechos como obligaciones por igual, sin distinción de sexo, ideología, creencias o costumbres. No obstante, a opinión de los autores esta definición no está acorde a lo que se pone en práctica actualmente, porque a más de existir diversidad cultural existe la interrelación entre ellas y se podría decir que coexisten entre sí.

\section{Multiculturalidad}

El termino multiculturalidad es un poco más alejado a lo que se vivencia actualmente, pero no podemos dejarlo de un lado porque en ciertos casos si se evidencia esas diferencias entre culturas y es necesario utilizar esta terminología. En cuanto a este concepto Higuera \& Castillo (2015) afirman que:

La multiculturalidad acepta la existencia de varias culturas en un mismo contexto y tiempo. Están unidas temporal y espacialmente, pero se da, al mismo tiempo, la suficiente lejanía (en cuanto a percepciones y actitudes) como para evitar la relación entre ellas. Esta situación llega a acentuar las diferencias y crear auténticos guetos, barrios aislados como un archipiélago. (pp. 5-6).

Sin embargo, este tipo de concepciones son las que han provocado enfoques de menosprecio hacia culturas distintas al comunizado mestizo. En contradicción a esta 
argumentación Ferrer (2014) en su artículo denominado multiculturalidad ecuatoriana e historia nacional dice que:

El hecho de que la Constitución ecuatoriana de 1998 reconociera el carácter multicultural y multiétnico del Estado abriría un nuevo camino para que un sector importante de la población nacional reivindicara su condición de indígena en el escenario político, en este contexto el hecho de ser considerados como indígenas lo que hasta ese momento se vivía como el estigma de un avasallamiento se vuelve algo valorizador y liberador, aunque susceptible de ser interpretado por algunos como un discurso separatista $\mathrm{y}$ tribalizador que, al privilegiar lo indígena, alienta el cultivo de esquemas impermeables de otredad. Por lo demás, el ejercicio de la crítica contribuye de modo positivo a la clarificación y el perfeccionamiento de los programas políticos. (p. 7)

En tal sentido, se evidencia que esta concepción es más problematizadora que las anteriores, los criterios de los autores denotan dos las variables que surgen. Por un lado, está la convivencia dentro de un mismo espacio y tiempo y por otro lado, una conceptualización de la multiculturalidad como una forma de revalorizar a los pueblos indígenas y de cierta manera introducirlos en la vida política y que de alguna manera esto se vea reflejado hoy en día en el sistema político.

En síntesis, las tres terminologías que a lo largo del tiempo han sido utilizadas y fortalecidas una más que otra, sin duda tienen esa distinción necesaria para ser utilizada cuando el contexto lo amerita. Sin embargo, a opinión de los autores se considera que la concepción que demuestra la interrelación cultural existente en el país es la Interculturalidad, puesto que actualmente las culturas son reconocidas y valorados por sus características distintivas, y estas están amparadas en la Constitución del país.

Krainer \& Guerra (2016) (citando a la constitución del 2008 mencionan que:

el Ecuador decidió construir una nueva forma de convivencia ciudadana, en diversidad y armonía con la naturaleza, para alcanzar el buen vivir, el sumak kawsay, como compromiso con el presente y el futuro. Por ello el Ecuador es un Estado 
constitucional de derechos y justicia, social, democrático, soberano, independiente, unitario, intercultural, plurinacional y laico (p. 1).

Por lo tanto, se puede decir que Ecuador es un país que posee una buena interrelación cultural, es decir, es intercultural, "país en donde conviven y comparten, junto con la cultura mestiza, 14 nacionalidades y 18 pueblos, hecho por el que se ha reconocido su condición pluricultural y multiétnica" (Hidalgo \& Erazo, 2017.p. 12). A partir de aquello se analiza su implicación en el ámbito educativo, puesto que al igual que sus definiciones variaron con el tiempo, su puesta en practica dentro de la educación no fue la excepción.

\section{Educación Intercultural en el Ecuador}

En Ecuador a partir del año 2008 con la actualización de la constitución de la república el país fue considerado como intercultural, plurinacional y multiétnico, y es apenas ahí cuando realmente se comenzó a desarrollar propuestas educativas que abarquen o se enmarquen dentro de la interculturalidad que ya definimos anteriormente, pero, ¿en qué medida esto se ha cumplido o no? Y será que ¿realmente estamos viviendo una interculturalidad?

Analizando esta situación, Ibáñez-Salgado et al., (2012) concluyen lo siguiente:

A nuestro juicio, la visión de interculturalidad predominante permanece anclada en una lógica tradicional, positivista, que se resiste a ser modificada, a pesar de los cambios paradigmáticos aceptados en el discurso educativo oficial de los países de la región. De este modo, la interculturalidad como propuesta pedagógica y ética se evidencia inmersa en una concepción general acerca del desarrollo que refleja la dimensión universalista del proyecto moderno, cuya pretensión de homogeneidad de lo humano constituye el marco epistémico desde el cual clasificamos a los estudiantes y categorizamos sus necesidades educativas y culturales. (pp. 217-218).

De esta manera se evidencia que las modificaciones que cada uno de estos grupos interculturales conllevan entre sí y de cierta manera adoptando y compartiendo las diferentes formas de vida y de formas culturales que cada grupo tiene y lo va 
desarrollando a lo largo de la vida; sin embargo es necesario también tener en cuenta el desarrollo cultural que cada uno de nosotros tenemos ya que de nada serviría si solamente mantenemos la mente cerrada hacia nuevas formas de analizar y visualizar el entorno cultural, educativo en el cual vivimos y nos lleva a relacionarnos con otras culturas que sin duda alguna tienen sus características bien marcadas y que juntos formamos una comunidad grande en donde todos tenemos los mismos derechos así como obligaciones que irlas desarrollando cada día.

Partiendo desde esta perspectiva podríamos determinar si justamente todos estos cambios y estas reformas educativas que se han venido desarrollando a lo largo de la historia de nuestro país han servido o solamente ha quedado plasmado en papeles en especial las reformas del sistema educativo con respecto al Sumak Kawsay o buen vivir. Pues bien, a opinión de los autores estos cambios o alcances interculturales en el sistema educativo se ha dado parcialmente, puesto que, desde las reformas se ha planteado una educación friccionada, con esto resaltamos el hecho de existir un currículo común que es utilizado especialmente en las zonas urbanas, y por el contrario en zonas rurales o escuelas bilingües se aplica el MOSEI, el cual si tiene un enfoque cultural.

\section{En concordancia con esto Rodríguez, (2019) menciona lo siguiente:}

Respecto al bilingüismo, puede decirse que en la generalidad de los casos estudiados sigue la misma lógica unidireccional de la "interculturalidad": son los "otros" indígenas los que deben aprender el castellano, la lengua de la sociedad hegemónica dominante, mientras que a la inversa las mallas curriculares del Sistema de Educación Nacional, el cual se presume intercultural, no han incorporado, en ningún caso, la enseñanza de al menos una de las lenguas indígenas existentes en el país. (p. 25).

Aunque se puede justificar que en las zonas rurales existe mayor población y representación de las diversas culturas y es donde se ubican las escuelas bilingües, no es un argumento para que exista esta fricción, porque hay que tener en cuenta que el auge de la migración hacia las ciudades ha provocado una mayor interrelación entre culturas también ya dentro de las cuidades que antes se consideraba era población de mestizos, y es ahí donde evidencia actualmente y de manera clara la interculturalidad. No obstante, se considera que el sistema educativo debe ser descolonizado, dejar atrás la vieja idea de 
que solo en el campo o en páramo están la culturas. Además, es importante mencionar que con el paso del tiempo y las investigaciones realizadas de ha podido evidenciar que las culturas tienen mucha información y conocimiento que aportar en la educación y sobre todo para construir una identidad en el país.

Rodríguez, (2017) reflexionando sobre esto menciona que:

Para lograr este cambio es fundamental la transformación del sistema educativo, de manera que la investigación en los establecimientos de educación superior (como universidades e institutos públicos) debe favorecer a la industria económica del Estado (Plan Nacional para el Buen Vivir 2009-2013). Sin embargo, y pese a que no se recoge en estos planes, las enseñanzas básicas y medias también deben perseguir el mismo objetivo. (p.74).

Como podemos darnos cuenta estas reformas en varias ocasiones han quedado solamente en papeles escritos debido a que en la vida cotidiana la realidad es diferente, sin embargo, también depende de cada uno fomentar estos principios y valores para lograr un estado más equitativo y lograr los fines del buen vivir que lo comentamos anteriormente. Además, es importante acotar que todos estos principios que rigen o están vigentes en la Constitución Política del Ecuador (2008), la Ley Orgánica de Educación Intercultural (2011) y el Reglamento General a la Ley Orgánica de educación Intercultural (2012) han ofrecido el soporte jurídico para la interculturalidad y la educación intercultural en el Ecuador. Sus artículos reflejan el deseo del estado ecuatoriano de trabajar a favor de la interculturalidad, y que depende de los docentes fomentar estos principios en las diferentes aulas de clase y sobre todo enseñar con el ejemplo.

Como se puede evidenciar existe diferentes perceptos hacia la diversidad de culturas y es un tema de mucha discusión, que además denota grandes desafíos como: la aceptación a que los diferentes pueblos y comunidades de nuestro territorio surjan como entes autónomos dentro de las diferentes cosmovisiones andinas; esto no ha sido fácil en su totalidad debido a diversas limitantes como la discriminación social, la falta de libertad de expresión, el poco apoyo al desarrollo de las representaciones culturales como: arte, teatro, danza, música, entre otras. Aunque poco a poco esto va cambiando aún es necesario ser consientes sobre el cómo estos pueblos han ido introduciéndose en esta 
forma de educación intercultural dentro de nuestro país y la gran riqueza en conocimientos ancestrales que esta población posee, puesto que "la educación intercultural logra incorporar una serie de elementos que determinan la práctica de la educación"(Espinoza \& Ley, 2020, p. 275).

\section{CONCLUSIONES}

A manera de conclusión de puede determinar que las diferentes terminologías utilizadas hoy en día para describir a la diversidad cultural, y diferenciar una de otra no hacen más que marcar cierta distancia entre los miembros de una comunidad, no obstante, el termino de interculturalidad es el que más representa la situación real de Ecuador puesto que a partir de la constitución del 2008, se promovió la interacción entre las diferentes culturas y se las concibió y consideró como poblaciones de igual índole. Eliminando cualquier situación de rechazo, aunque en su totalidad esto no se ha logrado y todavía se denotan de cierta manera el distanciamiento entre culturas.

En cuanto a la interculturalidad en la educación a partir de las reformas educativas que se han desarrollado con el paso del tiempo en nuestro país aún no han sido llevados a la vida cotidiana en su totalidad. Por ejemplo, existe distinciones entre una institución particular a una pública porque aún se ve reflejado la discriminación social y esto se ve reflejado en los estudiantes que a veces piensan que por ser publica la enseñanza es más deficiente que en la privada, y por otra parte está el uso del MOSEI en instituciones bilingües y el currículo común en las otras instituciones, lo cual sin duda debe ser subsanado para que exista realmente una educación intercultural en el país. En definitiva:

Aun cuando la población ecuatoriana se caracteriza por esta enorme riqueza, la educación que se ha ofrecido a los pueblos indígenas ha estado orientada tradicionalmente a promover su asimilación indiscriminada, lo que ha contribuido a limitar su desarrollo sociocultural y económico. (Conejo, 2008, p. 64)

Finalmente, se puede mencionar que en cuanto a la consecución del buen vivir depende de todos no solamente de autoridades ni docentes, sino también de estudiantes y padres de familia es decir de toda la sociedad, aceptando la diversidad cultural, viviendo en armonía y sin discriminación o rechazo alguno, de tal forma que esto no solamente quede en artículos en la constitución sino sea llevado a la práctica diaria, en definitiva, "se 
requiere activar un diseño y redirección estratégica en el ámbito de la formación intercultural" (E. Espinoza Freire et al., 2019, p. 341).

\section{REFERENCIAS BIBLIOGRÁFICAS}

Bernabé, M. (2012). Pluriculturalidad, multiculturalidad e interculturalidad, conocimientos necesarios para la labor docente. Revista Educativa Hekademos, $11,67-76$.

Conejo, A. (2008). Educación Intercultural Bilingüe en el Ecuador: La propuesta educativa y su proceso. Alteridad: Revista de Educación, 3(2), 64-82. https://dialnet.unirioja.es/servlet/articulo?codigo=5981149

Espinoza Freire, E., Castellano Gil, J. M., \& Herrera Montero, L. A. (2019). La dimensión intercultural en la formación docente en Ecuador. Psychology, Society \& Education, 11(3), 341-354. https://dialnet.unirioja.es/servlet/articulo? codigo=7283613

Espinoza Freire, E. E., \& Ley Leyva, N. V. (2020). Educación intercultural en el Ecuador: una revisión sistemática. Revista de Ciencias Sociales, 26(2), 275-288. https://dialnet.unirioja.es/servlet/articulo?codigo=7599945\&info=resumen\&idio $\mathrm{ma}=\mathrm{ENG}$

Ferrer Muñoz, M. (2014). MULTICULTURALIDAD ECUATORIANA E HISTORIA NACIONAL. Revista Castellano-Manchega de Ciencias Sociales, 17, 215-227. http://www.redalyc.org/articulo.oa?id=322132552015

Hidalgo Mantilla, G. D., \& Erazo Ortega, M. P. (2017). DECLARACIÓN, RACIONALIZACIÓN Y VIVENCIA DE LA EDUCACIÓN INTERCULTURAL BILINGÜE EN EL ECUADOR. Revista Inclusiones, 4(4), 12-26. https://revistainclusiones.org/index.php/inclu/article/view/641

Higuera, É., \& Castillo, N. (2015). LA INTERCULTURALIDAD COMO DESAFÍO PARA LA EDUCACIÓN ECUATORIANA. Sophia: Colección de Filosofía de La Educación, 18(1), 147-162. https://doi.org/10.17163/soph.n18.2015.08

Ibáñez-Salgado, N., Díaz-Arce, T., Druker-Ibáñez, S., \& Rodríguez-Olea, M. (2012). La comprensión de la diversidad en interculturalidad y educación. CONVERGENCIA. Revista de Ciencias Sociales, 59, 215-240. http://www.scielo.org.mx/scielo.php?script=sci_arttext\&pid=S140514352012000200009 
Krainer, A., \& Guerra, M. (2016). Interculturalidad y educación. Desafíos docentes. Unidad Editorial de FLACSO Ecuador. https://biblio.flacsoandes.edu.ec/catalog/resGet.php?resId=56204

Rodríguez Cruz, M. (2017). Interculturalidad, plurinacionalidad y sumak kawsay en Ecuador. La construcción de un nuevo modelo de Estado a través de la educación intercultural bilingüe: discurso y realidad. Perfiles Educativos, XXXIX(157), 7086. http://www.scielo.org.mx/scielo.php?script=sci_arttext\&pid=S018526982017000300070

Rodríguez Cruz, M. (2019). Interculturalidad y Educación Intercultural Bilingüe.: Vacíos y Desafíos en Ecuador. In M. Osuna Rodríguez \& M. Rivera Mateos (Eds.), VII Premio Intercultural "Luis Rodríguez" de investigación e innovación sobre interculturalidad (pp. 7-31). UCO PRESS (Universidad de Córdoba). https://dialnet.unirioja.es/servlet/articulo?codigo=7011158

UNESCO. (n.d.). Interculturalidad . Retrieved October 25, 2021, from https://es.unesco.org/creativity/interculturalidad 